TRANSACTIONS OF THE

AMERICAN MATHEMATICAL SOCIETY

Volume 363, Number 12, December 2011, Pages 6197-6218

S 0002-9947(2011)05596-0

Article electronically published on July 26, 2011

\title{
TWO-POINT DISTORTION THEOREMS FOR HARMONIC AND PLURIHARMONIC MAPPINGS
}

\author{
PETER DUREN, HIDETAKA HAMADA, AND GABRIELA KOHR
}

\begin{abstract}
Two-point distortion theorems are obtained for affine and linearly invariant families of harmonic mappings on the unit disk, with generalizations to pluriharmonic mappings of the unit ball in $\mathbb{C}^{n}$. In particular, necessary and sufficient conditions are given for a locally univalent harmonic or pluriharmonic mapping to be univalent. Some particular subclasses are also considered.
\end{abstract}

\section{INTRODUCTION}

The purpose of this paper is to develop some two-point distortion theorems for harmonic mappings in the plane, with extensions to pluriharmonic mappings in $\mathbb{C}^{n}$. Our results are generalizations of known distortion theorems for analytic functions, which we now proceed to describe.

For the class $S$ of analytic univalent functions in the unit disk $U$, normalized by $f(0)=0$ and $f^{\prime}(0)=1$, Koebe's classical distortion theorem gives the estimate $|f(z)| \geq|z|(1+|z|)^{-2}$, which can be viewed as a sharp lower bound on the distance from $f(0)$ to $f(z)$ in terms of the distance from 0 to $z$. Some years ago, Blatter [1] found a general two-point distortion theorem that is both necessary and sufficient for univalence and requires no normalization. His result is formulated in terms of the hyperbolic metric

$$
d(a, b)=\frac{1}{2} \log \frac{1+\rho(a, b)}{1-\rho(a, b)}, \quad \text { where } \quad \rho(a, b)=\left|\frac{a-b}{1-\bar{a} b}\right|, \quad a, b \in U,
$$

and $D_{1} f(z)=\left(1-|z|^{2}\right) f^{\prime}(z)$. Here is Blatter's theorem.

Theorem A. If $f$ is analytic and univalent in $U$, then

$$
|f(a)-f(b)|^{2} \geq \frac{\sinh ^{2}(2 d(a, b))}{8 \cosh (4 d(a, b))}\left(\left|D_{1} f(a)\right|^{2}+\left|D_{1} f(b)\right|^{2}\right), \quad a, b \in U .
$$

Conversely, if a nonconstant analytic function $f$ satisfies this inequality for all $a, b \in U$, then $f$ is univalent in $U$.

Received by the editors August 7, 2009.

2010 Mathematics Subject Classification. Primary 32H02; Secondary 30C45.

Key words and phrases. Harmonic mapping, pluriharmonic mapping, two-point distortion, affine invariance, linear invariance, univalence, convex mapping, close-to-convex mapping, starlike mapping.

The second author was partially supported by Grant-in-Aid for Scientific Research (C) No. 22540213 from Japan Society for the Promotion of Science, 2011.

The third author was supported by the UEFISCSU-CNCSIS Grant PN-II-ID 524/2007. 
Kim and Minda [15] observed that with very little additional effort the Koebe distortion theorem can be strengthened to an invariant form that is both necessary and sufficient for univalence. They obtained the following result.

Theorem B. If $f$ is analytic and univalent in $U$, then

$$
|f(a)-f(b)| \geq \frac{\sinh (2 d(a, b))}{2 \exp (2 d(a, b))} \max \left\{\left|D_{1} f(a)\right|,\left|D_{1} f(b)\right|\right\}, \quad a, b \in U .
$$

Conversely, if a nonconstant analytic function $f$ satisfies this inequality for all $a, b \in U$, then $f$ is univalent in $U$.

The keys to Blatter's proof of Theorem A are some standard coefficient estimates for univalent functions and a special inequality for solutions to a certain nonlinear differential equation. Kim and Minda [15] adapted Blatter's argument to embed the result in a one-parameter family of sharp two-point distortion theorems. Jenkins 13 then applied his General Coefficient Theorem to extend the result of Kim and Minda to a wider range of parameters and also to obtain an upper bound, thus culminating in the following theorem.

Theorem C. If $f$ is analytic and univalent in $U$, then for each $p>0$,

$$
|f(a)-f(b)| \leq \frac{1}{2} \sinh (2 d(a, b))\left(\frac{\left|D_{1} f(a)\right|^{p}+\left|D_{1} f(b)\right|^{p}}{2}\right)^{1 / p}, \quad a, b \in U .
$$

Moreover, if $p \geq 1$, then

$$
|f(a)-f(b)| \geq \frac{\sinh (2 d(a, b))}{2[\cosh (2 p d(a, b))]^{1 / p}}\left(\frac{\left|D_{1} f(a)\right|^{p}+\left|D_{1} f(b)\right|^{p}}{2}\right)^{1 / p}, \quad a, b \in U .
$$

Conversely, if a nonconstant analytic function $f$ satisfies the inequality (4) for some $p>0$ and for all $a, b \in U$, then $f$ is univalent in $U$.

It may be observed that for $p=2$ the inequality (4) is Blatter's theorem (Theorem A). The limiting case of (4) as $p \rightarrow \infty$ is the invariant form of the Koebe distortion theorem (Theorem B).

Kraus and Roth [18] recently applied the Goluzin inequalities to obtain a family of sharp two-point distortion theorems analogous to Theorem $\mathrm{C}$ for negative values of the parameter $p$. Their result can be stated as follows.

Theorem D. If $f$ is analytic and univalent in $U$, then for each $p<0$,

$$
|f(a)-f(b)| \geq \tanh (d(a, b))\left(\frac{\left|D_{1} f(a)\right|^{p}+\left|D_{1} f(b)\right|^{p}}{2}\right)^{1 / p}, \quad a, b \in U
$$

and

(6)

$$
|f(a)-f(b)| \leq \frac{\sinh (2 d(a, b))}{2[\cosh (2 p d(a, b))]^{1 / p}}\left(\frac{\left|D_{1} f(a)\right|^{p}+\left|D_{1} f(b)\right|^{p}}{2}\right)^{1 / p}, \quad a, b \in U .
$$

Conversely, if a nonconstant analytic function $f$ satisfies the inequality (5) for some $p<0$ and for all $a, b \in U$, then $f$ is univalent in $U$.

Other two-point distortion results for analytic univalent functions can be found in [7, 10, [13, 14], [18, 20], 21], 22, 32], 34], 35]. 
In this paper we generalize the above theorems to complex-valued harmonic functions, or harmonic mappings of the disk. Here the basic tool, playing the role of the Koebe distortion theorem for analytic univalent functions, will be a growth theorem for affine and linearly invariant families of harmonic mappings, due to Sheil-Small [37]. Sheil-Small's theorem will be recorded in the next section, together with background material on harmonic mappings. Our results provide necessary and sufficient conditions for univalence of sense-preserving harmonic mappings of the unit disk. In the second part of the paper we develop versions of these results for pluriharmonic mappings of the unit ball in $\mathbb{C}^{n}$.

\section{Preliminaries}

Let $\Omega$ be a domain in the complex plane $\mathbb{C}$, and let $f$ be a complex-valued function of class $C^{1}$ in $\Omega$. The Jacobian of $f$ is given by

$$
J_{f}=\left|\frac{\partial f}{\partial z}\right|^{2}-\left|\frac{\partial f}{\partial \bar{z}}\right|^{2}
$$

It is well known that $f$ is locally univalent if $J_{f}(z) \neq 0$ in $\Omega$ and that the converse is also true if $f$ is analytic. A theorem of Lewy [19] asserts that the converse remains true for harmonic mappings in the plane. Thus a locally univalent harmonic mapping is either sense-preserving (if $J_{f}(z)>0$ in $\Omega$ ) or sense-reversing (if $J_{f}(z)<$ $0)$. A harmonic mapping of the unit disk $U$ has the unique representation $f=$ $h+\bar{g}$, where $h$ and $g$ are analytic in $U$ and $g(0)=0$. This is called the canonical representation of $f$. Note that $f$ is sense-preserving if and only if $\left|g^{\prime}(z)\right|<\left|h^{\prime}(z)\right|$ for all $z \in U$. This implies that $h^{\prime}(z) \neq 0$ in $U$, so that $h$ is locally univalent.

Let $\mathcal{H}(U)$ be the set of analytic functions in $U$. Let $S_{H}$ denote the family of sense-preserving univalent harmonic mappings $f=h+\bar{g}$ on $U$, where $h, g \in \mathcal{H}(U)$ and are normalized by $h(0)=0, h^{\prime}(0)=1$, and $g(0)=0$. Let $S_{H}^{0}$ denote the subclass of $S_{H}$ with the further normalization $g^{\prime}(0)=0$. The family $S_{H}$ is normal, and $S_{H}^{0}$ is compact (see [3] or [5]).

We will be concerned with families of harmonic mappings that are both linearly invariant and affine invariant. Linear invariance was first studied by Pommerenke 30. for families of locally univalent analytic functions. Sheil-Small [37] then generalized the notion to families of harmonic mappings. A family $\mathcal{F} \subset S_{H}$ of harmonic mappings is said to be linearly invariant if $f=h+\bar{g} \in \mathcal{F}$ implies that

$$
\frac{f\left(\left(z+z_{0}\right) /\left(1+\overline{z_{0}} z\right)\right)-f\left(z_{0}\right)}{\left(1-\left|z_{0}\right|^{2}\right) h^{\prime}\left(z_{0}\right)} \in \mathcal{F} \quad \text { for each } z_{0} \in U .
$$

The family $\mathcal{F}$ is affine invariant if $f \in \mathcal{F}$ implies that

$$
\frac{f(z)+\varepsilon \overline{f(z)}}{1+\varepsilon g^{\prime}(0)} \in \mathcal{F} \quad \text { for each } \varepsilon \in U .
$$

The full family $S_{H}$ is both linearly and affine invariant.

The order of a family $\mathcal{F} \subset S_{H}$ is defined by

$$
\alpha=\alpha(\mathcal{F})=\sup \left\{\frac{1}{2}\left|h^{\prime \prime}(0)\right|: f=h+\bar{g} \in \mathcal{F}\right\} .
$$

In view of the maximum principle and the fact that $h$ is locally univalent, we see that $\alpha(\mathcal{F}) \geq 1$ (cf. 30] $)$. Bieberbach's theorem says that $\alpha(S)=2$. It has long been conjectured that $\alpha\left(S_{H}\right)=3$, but this is still an open question. 
For any family $\mathcal{F} \subset S_{H}$, let $\mathcal{F}^{0}=\mathcal{F} \cap S_{H}^{0}$, the subfamily of functions $f=h+\bar{g} \in \mathcal{F}$ for which $g^{\prime}(0)=0$. For later reference we now record the basic growth theorem for subfamilies of $S_{H}$, due to Sheil-Small [37] (see also [5], p. 97).

Theorem E. Let $\mathcal{F} \subset S_{H}$ be an affine and linearly invariant family of order $\alpha$. Then each function $f \in \mathcal{F}^{0}$ satisfies the inequalities

$$
\frac{1}{2 \alpha}\left[1-\left(\frac{1-r}{1+r}\right)^{\alpha}\right] \leq|f(z)| \leq \frac{1}{2 \alpha}\left[\left(\frac{1+r}{1-r}\right)^{\alpha}-1\right], \quad r=|z|<1 .
$$

Actually, a careful examination of the proof reveals that the upper bound in (7) is valid more generally for locally univalent, sense-preserving harmonic mappings $f=h+\bar{g}$ that are normalized by $f(0)=0, h^{\prime}(0)=1$, and $g^{\prime}(0)=0$. In other words, $f$ need not be globally univalent.

For a function $f=h+\bar{g}$ harmonic in $U$, the two quantities

$$
Q(z)=\left(1-|z|^{2}\right)\left(\left|h^{\prime}(z)\right|+\left|g^{\prime}(z)\right|\right) \quad \text { and } \quad R(z)=\left(1-|z|^{2}\right)\left(\left|h^{\prime}(z)\right|-\left|g^{\prime}(z)\right|\right)
$$

will play the role of the weighted derivative $D_{1} f$. Note that if $f \in \mathcal{H}(U)$, then $Q(z)=R(z)=\left|D_{1} f(z)\right|$.

\section{Distortion OF HARMONIC MAPPINGS}

We begin with a two-point distortion theorem for harmonic mappings that is a generalization of Theorem B, the invariant form of the Koebe distortion theorem for analytic functions as proved by Kim and Minda [15. The proof is similar to that of Kim and Minda in that it uses linear and affine invariance to convert the basic distortion theorem (Theorem E) into invariant form. The same argument gives an upper bound for distortion as well as a lower bound. The lower estimate was also obtained by Pfaltzgraff [26] and is a generalization of a result in [10] (Theorem 4).

Theorem 1. Let $\mathcal{F} \subset S_{H}$ be an affine and linearly invariant family of order $\alpha$. Then each $f \in \mathcal{F}$ satisfies the inequalities

$$
|f(a)-f(b)| \geq \frac{1}{2 \alpha}[1-\exp (-2 \alpha d(a, b))] \max \{R(a), R(b)\}, \quad a, b \in U
$$

and

$$
|f(a)-f(b)| \leq \frac{1}{2 \alpha}[\exp (2 \alpha d(a, b))-1] \min \{Q(a), Q(b)\}, \quad a, b \in U .
$$

Conversely, if a harmonic mapping $f$ has nonnegative Jacobian that does not vanish everywhere in $U$, and if $f$ satisfies the inequality (8) for some $\alpha>0$ and all pairs of points $a, b \in U$, then $f$ is univalent in $U$.

Proof. Fix a pair of distinct points $a, b \in U$ and define the Möbius transformation

$$
\varphi(z)=\frac{z+a}{1+\bar{a} z} .
$$

If $f=h+\bar{g} \in \mathcal{F}$, then by linear invariance the function

$$
F(z)=\frac{f(\varphi(z))-f(a)}{\left(1-|a|^{2}\right) h^{\prime}(a)}=H(z)+\overline{G(z)}
$$

also belongs to $\mathcal{F}$. A calculation shows that $G^{\prime}(0)=g^{\prime}(a) / \overline{h^{\prime}(a)}$, and so $\varepsilon=G^{\prime}(0) \in$ $U$ since $\left|g^{\prime}(a)\right|<\left|h^{\prime}(a)\right|$. Hence by affine invariance of $\mathcal{F}$ the function

$$
F_{0}(z)=\frac{F(z)-\bar{\varepsilon} \overline{F(z)}}{1-|\varepsilon|^{2}}
$$


belongs to $\mathcal{F}^{0}$ and therefore satisfies the conclusion of Theorem E. This says that

$$
\frac{1}{2 \alpha}[1-\exp (-2 \alpha d(0, z))] \leq\left|F_{0}(z)\right| \leq \frac{1}{2 \alpha}[\exp (2 \alpha d(0, z))-1],
$$

where the upper and lower bounds in (7) have been expressed in terms of the hyperbolic metric. Now recall that the hyperbolic metric is Möbius invariant, so that $d(0, z)=d\left(\varphi^{-1}(a), \varphi^{-1}(b)\right)=d(a, b)$ if we choose $z=\varphi^{-1}(b)$. Thus we find that

$$
\frac{1}{2 \alpha}[1-\exp (-2 \alpha d(a, b))] \leq\left|F_{0}\left(\varphi^{-1}(b)\right)\right| \leq \frac{1}{2 \alpha}[\exp (2 \alpha d(a, b))-1] .
$$

A straightforward calculation gives

$$
F_{0}\left(\varphi^{-1}(b)\right)=\frac{\overline{h^{\prime}(a)}(f(b)-f(a))-\overline{g^{\prime}(a)}(\overline{f(b)}-\overline{f(a)})}{\left(1-|a|^{2}\right)\left(\left|h^{\prime}(a)\right|^{2}-\left|g^{\prime}(a)\right|^{2}\right)},
$$

and the desired result follows by applying the triangle inequality to the numerator, then interchanging the roles of $a$ and $b$.

Conversely, suppose that a harmonic mapping $f$ satisfies (8) and has Jacobian $J_{f}(z)=\left|h^{\prime}(z)\right|^{2}-\left|g^{\prime}(z)\right|^{2} \geq 0$, but $J_{f}(z) \not \equiv 0$. Then its dilatation $\omega=g^{\prime} / h^{\prime}$ is analytic and has the property $|\omega(z)| \leq 1$ wherever $h^{\prime}(z) \neq 0$. Since $\left|g^{\prime}(z)\right| \leq\left|h^{\prime}(z)\right|$ and $J_{f}(z) \not \equiv 0$, it is clear that $h^{\prime}(z)$ cannot vanish identically and so its zeros are isolated. Any such zero is a removable singularity of $\omega$, since $|\omega(z)| \leq 1$ in some deleted neighborhood of that point. Thus $\omega$ is analytic and $|\omega(z)| \leq 1$ for all $z \in U$. If $f$ is not univalent, then $f(a)=f(b)$ for some $a, b \in U$ with $a \neq b$. Then it follows from (8) that $J_{f}(a)=J_{f}(b)=0$. Consequently, $f$ is not locally univalent at $a$ (or at $b$ ) and so $f\left(a_{k}\right)=f\left(b_{k}\right)$ for some sequences $\left\{a_{k}\right\}$ and $\left\{b_{k}\right\}$ tending to $a$, with $a_{k} \neq b_{k}$. In view of $(8)$, this implies that $J_{f}\left(a_{k}\right)=0$ for all $k$. Without loss of generality we may assume that all points $a_{k}$ are different from $a$. There are now two cases. If $h^{\prime}\left(a_{k}\right)=g^{\prime}\left(a_{k}\right)=0$ for all $k$, then $h^{\prime}(z) \equiv g^{\prime}(z) \equiv 0$ and so $J_{f}(z) \equiv 0$, contrary to assumption. If on the other hand, $h^{\prime}\left(a_{k}\right) \neq 0$ for some $k$, then $\left|\omega\left(a_{k}\right)\right|=1$ and the maximum modulus principle implies that $\omega(z) \equiv e^{i \theta}$, a unimodular constant. In other words, $g^{\prime}(z)=e^{i \theta} h^{\prime}(z)$ in $U$, which again implies that $J_{f}(z) \equiv 0$. The conclusion is that if $f$ satisfies (8) for all $a, b \in U$, and if $J_{f}(z) \geq 0$ but $J_{f}(z) \not \equiv 0$, then $f$ is univalent in $U$.

It should be remarked that the upper bound (9) does not actually require univalence but remains valid if $f$ is only locally univalent, since the proof is based on the upper bound in (7). (See the remark following the statement of Theorem E.)

The theorem applies in particular to the subfamily of convex mappings in $S_{H}$, which is affine and linearly invariant and has order $\alpha=2$. (See [3] or [5].) According to Theorem 1 , any such mapping $f$ will satisfy the two-point distortion inequalities (8) and (9) with $\alpha=2$. Similarly, the subfamily of $S_{H}$ consisting of close-to-convex mappings is affine and linearly invariant and has order $\alpha=3$ (see [3] or [5]). Thus by Theorem 1 any such mapping $f$ must satisfy (8) and (9) with $\alpha=3$. The theorem does not apply directly to the subfamily of mappings that are starlike with respect to the origin, since this property is not linearly invariant. However, since every starlike mapping is close-to-convex, the same inequalities hold, with $\alpha=3$, for starlike mappings $f \in S_{H}$.

In fact, when $\alpha=3$ the inequalities (8) and (9) are sharp for starlike mappings in $S_{H}$. To see this we have only to note that the upper and lower bounds in (7) are attained by suitable rotations of the harmonic Koebe function, a starlike mapping 
in the class $S_{H}^{0}$. (See [5], p. 97.) On the other hand, when $\alpha=2$, the lower bound in (8) is not sharp for convex mappings, since the lower bound in (7) is not sharp in this case. The sharp bounds on $|f(z)|$ for convex mappings $f \in S_{H}^{0}$ are not known (cf. [5], p. 100).

Theorem 1 applies to the full family $S_{H}$ but does not provide useful information, because the exact order of this linearly invariant family is unknown. It is conjectured, however, that $\alpha\left(S_{H}\right)=3$. If this were true, the sharp bounds on growth of functions $f \in S_{H}^{0}$ would be given by (7) with $\alpha=3$. The two-point distortion bounds (8) and (9) would then follow (with $\alpha=3$ ) for all mappings $f \in S_{H}$ and would be sharp. But at present this is only a conjecture, since the best known bound on $\alpha\left(S_{H}\right)$ is around 50 (see [5], p. 96).

On the other hand, it can be proved by the method of extremal length (see [3], Theorem 4.4 or [5], p. 92) that each function $f \in S_{H}^{0}$ satisfies the inequality

$$
|f(z)| \geq \frac{1}{4} \frac{r}{(1+r)^{2}}, \quad r=|z|<1,
$$

which can be taken as an analogue of the Koebe distortion theorem. Starting with (10) and using arguments similar to those in the proof of Theorem 1, we can establish the following theorem.

Theorem 2. Each $f \in S_{H}$ satisfies the inequality

$$
|f(a)-f(b)| \geq \frac{1}{16}[1-\exp (-4 d(a, b))] \max \{R(a), R(b)\}, \quad a, b \in U .
$$

Conversely, if a harmonic mapping $f$ has Jacobian $J_{f}(z) \geq 0$ but $J_{f}(z) \not \equiv 0$, and if $f$ satisfies the inequality (11) for all pairs of points $a, b \in U$, then $f$ is univalent in $U$.

We conclude this section with two additional two-point distortion theorems for convex harmonic mappings, analogous to and derived from Theorems $\mathrm{C}$ and $\mathrm{D}$, respectively, for analytic functions. Here is the analogue of Theorem C.

Theorem 3. If $f$ is a convex harmonic mapping of class $S_{H}$, then for each $p>0$,

$$
|f(a)-f(b)| \leq \frac{1}{2} \sinh (2 d(a, b))\left(\frac{Q(a)^{p}+Q(b)^{p}}{2}\right)^{1 / p}, \quad a, b \in U .
$$

Moreover, if $p \geq 1$, then

$$
|f(a)-f(b)| \geq \frac{\sinh (2 d(a, b))}{2[\cosh (2 p d(a, b))]^{1 / p}}\left(\frac{R(a)^{p}+R(b)^{p}}{2}\right)^{1 / p}, \quad a, b \in U .
$$

Conversely, if a harmonic mapping $f$ has Jacobian $J_{f}(z) \geq 0$ but $J_{f}(z) \not \equiv 0$, and if $f$ satisfies the inequality (13) for some $p>0$ and for all $a, b \in U$, then $f$ is univalent in $U$.

Proof. Let us first consider the inequality (13). If $f=h+\bar{g}$ is a convex harmonic mapping in $S_{H}$, then the analytic function $\Phi_{\theta}=h+e^{i \theta} g$ is known to be univalent (in fact, convex in one direction) for each $\theta \in[0,2 \pi$ ) (see [3] or [5]). Thus for each $\theta \in[0,2 \pi)$ and $p \geq 1$ it follows from Theorem $\mathrm{C}$ that

$$
\left|\Phi_{\theta}(a)-\Phi_{\theta}(b)\right| \geq \frac{\sinh (2 d(a, b))}{2[\cosh (2 p d(a, b))]^{1 / p}}\left(\frac{\left|D_{1} \Phi_{\theta}(a)\right|^{p}+\left|D_{1} \Phi_{\theta}(b)\right|^{p}}{2}\right)^{1 / p}
$$


for all $a, b \in U$. For fixed $a, b \in U$ with $a \neq b$, there are now two cases. If $g(a)=g(b)$, then $\Phi_{\theta}(a)-\Phi_{\theta}(b)=f(a)-f(b)$ for every $\theta \in[0,2 \pi)$. If $g(a) \neq g(b)$, choose $\theta \in[0,2 \pi)$ so that

$$
e^{i \theta}=\frac{\overline{g(a)}-\overline{g(b)}}{g(a)-g(b)} .
$$

Then again $\Phi_{\theta}(a)-\Phi_{\theta}(b)=f(a)-f(b)$. On the other hand,

$$
\left|D_{1} \Phi_{\theta}(z)\right| \geq\left(1-|z|^{2}\right)\left(\left|h^{\prime}(z)\right|-\left|g^{\prime}(z)\right|\right)=R(z), \quad z \in U .
$$

Combining this inequality with (14), we obtain in either case the desired result (13). The relation (12) is proved in a similar way. To see that (13) implies the univalence of $f$ if $J_{f}(z) \geq 0$ but $J_{f}(z) \not \equiv 0$, we may adapt the argument used in the proof of Theorem 1 .

It would be interesting to extend the inequality (13), or to find an analogous result, for $0<p<1$.

It can be shown ( $c f$. 15) that the right-hand side of (13) is a decreasing function of $p$ for $p \geq 1$ and the right-hand side of (12) is an increasing function of $p$ for $p>0$. Hence the strongest form of Theorem 3 is obtained by taking $p=1$ in (13) and letting $p \rightarrow 0$ in (12). In this way we arrive at the following corollary.

Corollary. If $f$ is a convex harmonic mapping of class $S_{H}$, then

$$
\frac{1}{4} \tanh (2 d(a, b))(R(a)+R(b)) \leq|f(a)-f(b)| \leq \frac{1}{2} \sinh (2 d(a, b)) \sqrt{Q(a) Q(b)}
$$

for all $a, b \in U$. Conversely, if a harmonic mapping $f$ has Jacobian $J_{f}(z) \geq 0$ but $J_{f}(z) \not \equiv$, and if $f$ satisfies the left-hand inequality above for all $a, b \in U$, then $f$ is univalent in $U$.

By a similar line of reasoning, Theorem D can be applied to give a corresponding result for convex harmonic mappings.

Theorem 4. If $f$ is a convex harmonic mapping of class $S_{H}$, then for each $p<0$,

$$
|f(a)-f(b)| \geq \tanh (d(a, b))\left(\frac{R(a)^{p}+R(b)^{p}}{2}\right)^{1 / p}, \quad a, b \in U
$$

and

$$
|f(a)-f(b)| \leq \frac{\sinh (2 d(a, b))}{2[\cosh (2 p d(a, b))]^{1 / p}}\left(\frac{Q(a)^{p}+Q(b)^{p}}{2}\right)^{1 / p}, \quad a, b \in U .
$$

Conversely, if a harmonic mapping $f$ has Jacobian $J_{f}(z) \geq 0$ but $J_{f}(z) \not \equiv 0$, and if $f$ satisfies the inequality (15) for some $p<0$ and for all $a, b \in U$, then $f$ is univalent in $U$.

Corollary. If $f$ is a convex harmonic mapping of class $S_{H}$, then

$$
\tanh (d(a, b)) \sqrt{R(a) R(b)} \leq|f(a)-f(b)| \leq \frac{1}{2} \sinh (2 d(a, b)) \sqrt{Q(a) Q(b)}
$$

for all $a, b \in U$. Conversely, if a harmonic mapping $f$ has Jacobian $J_{f}(z) \geq 0$ but $J_{f}(z) \not \equiv 0$, and if $f$ satisfies the left-hand inequality above for all $a, b \in U$, then $f$ is univalent in $U$. 
The following lemma is needed to deduce the corollary from Theorem 4 . It implies that the strongest form of Theorem 4 is obtained by letting $p \rightarrow 0$ in (15) and (16).

Lemma 1. The right-hand side of (15) is an increasing function of $p$ for $p<0$, and the right-hand side of (16) is a decreasing function of $p$ for $p<0$.

Proof. Straightforward differentiation shows that the right-hand side of (15) is an increasing function of $p$ for $p<0$. Specifically, we may calculate the logarithmic derivative, multiply by $p^{2}$, then deduce that the resulting expression is positive for $p<0$ by differentiating again to see that it decreases to zero. To see that the right-hand side of (16) is a decreasing function of $p<0$, we follow an argument similar to that in [18]. With the notation $t=-p, A=e^{2 d(a, b)}, B=e^{-2 d(a, b)}$, $C=1 / Q(a)$, and $D=1 / Q(b)$, we can write

$$
\left(\frac{Q(a)^{p}+Q(b)^{p}}{2(\cosh (2 p d(a, b)))}\right)^{1 / p}=\left(\frac{A^{t}+B^{t}}{C^{t}+D^{t}}\right)^{1 / t}=r(t) .
$$

The problem is then to prove that the function $r(t)$ is increasing on $(0, \infty)$. We may assume that $C \geq D$. By a result of Marshall, Olkin, and Proschan [23, the function $r(t)$ is increasing if $A>0, B>0, C \geq D>0$, and $C / A \leq D / B$; that is, if $Q(a) \leq Q(b)$ and

$$
Q(b) \leq e^{4 d(a, b)} Q(a), \quad a, b \in U
$$

To prove that (17) holds, let $f=h+\bar{g}$ be an arbitrary convex function in $S_{H}$. Then as in the proof of Theorem 3, the function $\Phi_{\theta}=h+e^{i \theta} g$ is univalent for each $\theta \in[0,2 \pi)$. Hence the Koebe distortion theorem (see [4] or 31]) gives

or

$$
\left|\frac{\Phi_{\theta}{ }^{\prime}(z)}{\Phi_{\theta}{ }^{\prime}(0)}\right| \leq \frac{1+|z|}{(1-|z|)^{3}}, \quad z \in U,
$$

$$
\left(1-|z|^{2}\right)\left|\Phi_{\theta}{ }^{\prime}(z)\right| \leq\left(\frac{1+|z|}{1-|z|}\right)^{2}\left|\Phi_{\theta}{ }^{\prime}(0)\right|, \quad z \in U,
$$

for all $\theta \in[0,2 \pi)$. With a suitable choice of $\theta$ the last inequality yields

$$
\left(1-|z|^{2}\right)\left(\left|h^{\prime}(z)\right|+\left|g^{\prime}(z)\right|\right) \leq e^{4 d(0, z)}\left(1+\left|g^{\prime}(0)\right|\right), \quad z \in U,
$$

which says that

$$
Q(z) \leq e^{4 d(0, z)} Q(0), \quad z \in U
$$

Finally, we apply the linear invariance of the class of convex mappings together with the Möbius invariance of the hyperbolic metric, as in the proof of Theorem 1, to arrive at the inequality (17). This completes the proof.

Some different kinds of two-point distortion theorems for harmonic mappings can be found in [2].

\section{FAMILIES OF PLURIHARMONiC MAPPINGS}

In this section we introduce the notions of affine and linear invariance for families of locally univalent pluriharmonic mappings, and we present some basic results related to these notions. The results will be applied in Section 5 to prove two-point distortion theorems. We begin by recalling some standard notation. 
As usual, $\mathbb{C}^{n}$ is the space of $n$ complex variables $z=\left(z_{1}, \ldots, z_{n}\right)$ with inner product $\langle z, w\rangle=\sum_{j=1}^{n} z_{j} \overline{w_{j}}$ and norm $\|z\|=\langle z, z\rangle^{1 / 2}$. The open ball $\left\{z \in \mathbb{C}^{n}\right.$ : $\|z\|<r\}$ is denoted by $B_{r}^{n}$. We write $B^{n}$ for the unit ball $B_{1}^{n}$. In the case of one complex variable, $B^{1}$ is the unit disk $U$.

We denote by $L\left(\mathbb{C}^{n}, \mathbb{C}^{m}\right)$ the space of continuous linear operators from $\mathbb{C}^{n}$ into $\mathbb{C}^{m}$ with the standard operator norm, and let $I_{n}$ be the identity operator in $L\left(\mathbb{C}^{n}, \mathbb{C}^{n}\right)$.

The set of holomorphic mappings from a domain $\Omega \subset \mathbb{C}^{n}$ into $\mathbb{C}^{n}$ is denoted by $\mathcal{H}(\Omega)$. When $\Omega$ contains the origin, we say that a mapping $f \in \mathcal{H}(\Omega)$ is normalized if $f(0)=0$ and $D f(0)=I_{n}$. The family of normalized biholomorphic mappings on $B^{n}$ will be denoted by $S\left(B^{n}\right)$. In the case of one complex variable, $S\left(B^{1}\right)$ is the usual family $S$ of normalized univalent functions on the unit disk $U$. We let $K\left(B^{n}\right)$ denote the subfamily of $S\left(B^{n}\right)$ consisting of convex mappings. $\operatorname{Aut}\left(B^{n}\right)$ is the set of biholomorphic automorphisms of $B^{n}$.

A mapping $f \in \mathcal{H}(\Omega)$ is known to be locally biholomorphic in $\Omega$ if and only if its determinant $\operatorname{det} D f(z) \neq 0$ for all $z \in \Omega$ (see for instance [17]). The family of all normalized locally biholomorphic mappings on $B^{n}$ will be denoted by $\mathcal{L} S\left(B^{n}\right)$.

A complex-valued function $f$ of class $C^{2}$ on $B^{n}$ is said to be pluriharmonic if its restriction to every complex line is harmonic. This happens if and only if $\frac{\partial^{2}}{\partial z_{j} \partial \overline{z_{k}}} f(z) \equiv 0$ in $B^{n}$ for all $j, k=1,2, \ldots, n$. A real-valued $C^{2}$ function $f$ on $B^{n}$ is pluriharmonic if and only if it is the real part of some holomorphic function on $B^{n}$. Every real-valued harmonic function on $U$ is the real part of a holomorphic function, but this is no longer true for functions on $B^{n}$. In other words, when $n>1$ the pluriharmonic functions form a proper subclass of the harmonic functions on $B^{n}$. Every pluriharmonic mapping $f: B^{n} \rightarrow \mathbb{C}^{n}$ can be written as $f=h+\bar{g}$, where $g$ and $h$ are holomorphic mappings of $B^{n}$ into $\mathbb{C}^{n}$, and this representation is unique if $g(0)=0$.

We now define a class of pluriharmonic mappings on $B^{n}$ that will serve as an analogue of the class $S_{H}$ on the unit disk. Recall that $S_{H}$ consists of the univalent sense-preserving harmonic mappings $f=h+\bar{g}$, where $h, g \in \mathcal{H}(U), h(0)=g(0)=0$, and $h^{\prime}(0)=1$. We define $S_{H}\left(B^{n}\right)$ to be the family of all univalent pluriharmonic mappings $f=h+\bar{g}$ on $B^{n}$, where $h, g \in \mathcal{H}\left(B^{n}\right), g(0)=0$, and $h \in \mathcal{L} S\left(B^{n}\right)$. Observe that a mapping $f \in S_{H}\left(B^{n}\right)$ is not required to be sense-preserving; that is, its real Jacobian (when $f$ is regarded as a mapping from $\mathbb{R}^{2 n}$ to $\mathbb{R}^{2 n}$ ) need not be positive. However, as we will see shortly (in Theorem 5), a natural generalization of the one-variable condition $\left|g^{\prime}(z)\right|<\left|h^{\prime}(z)\right|$ is sufficient for $f$ to be sense-preserving. Note that every biholomorphic mapping is sense-preserving because its real Jacobian is equal to $|\operatorname{det} D f(z)|^{2}>0$.

The symbol $\mathcal{L} S_{H}\left(B^{n}\right)$ will denote the family of all pluriharmonic mappings $f=$ $h+\bar{g}$ on $B^{n}$ with $h \in \mathcal{L} S\left(B^{n}\right)$ and $g(0)=0$. Thus $S_{H}\left(B^{n}\right)$ is the family of univalent pluriharmonic mappings $f \in \mathcal{L} S_{H}\left(B^{n}\right)$. Let $S_{H}^{0}\left(B^{n}\right)$ be the subfamily of mappings $f=h+\bar{g} \in S_{H}\left(B^{n}\right)$ such that $D g(0)=0$. In contrast with the one-variable situation, neither $S_{H}\left(B^{n}\right)$ nor $S_{H}^{0}\left(B^{n}\right)$ is a normal family when $n>1$. For a proof, simply observe that $S\left(B^{n}\right) \subset S_{H}^{0}\left(B^{n}\right) \subset S_{H}\left(B^{n}\right)$ and $S\left(B^{n}\right)$ is not a normal family in dimension $n>1$ (see e.g. [8] and [9]).

To motivate the next theorem, recall that $f=h+\bar{g}$ is a sense-preserving harmonic mapping in the plane when $\left|h^{\prime}(z)\right|-\left|g^{\prime}(z)\right|>0$ for all $z \in U$, which implies that the analytic function $h+a g$ is locally univalent in $U$ for each $a \in \mathbb{C}$ with $|a|<1$. 
As we will now show, an appropriate generalization of this sense-preserving condition implies that a pluriharmonic mapping $f=h+\bar{g}$ of class $\mathcal{L} S_{H}\left(B^{n}\right)$ is actually sense-preserving and has the analogous property that $h+A g$ is locally biholomorphic in $B^{n}$ for linear operators $A$ of norm $\|A\|<1$.

Theorem 5. Let $f=h+\bar{g} \in \mathcal{L} S_{H}\left(B^{n}\right)$. If $\left\|D g(z)[D h(z)]^{-1}\right\|<1$ for $z \in B^{n}$, then $h+A g$ is locally biholomorphic in $B^{n}$ for each $A \in L\left(\mathbb{C}^{n}, \mathbb{C}^{n}\right)$ with $\|A\|<1$. Furthermore, $f$ is a sense-preserving mapping.

Proof. The hypothesis implies $\operatorname{det}\left(I_{n}+A D g(z)[D h(z)]^{-1}\right) \neq 0$ for $A \in L\left(\mathbb{C}^{n}, \mathbb{C}^{n}\right)$ with norm $\|A\|<1$ and for all $z \in B^{n}$. It follows that $\operatorname{det}(\operatorname{Dh}(z)+A D g(z)) \neq 0$, so that $h+A g$ is locally biholomorphic in $B^{n}$.

To see that $f$ is sense-preserving, recall that the real Jacobian of $f$ is

$$
J_{f}(z)=\operatorname{det}\left(\begin{array}{cc}
D h(z) & \overline{D g(z)} \\
D g(z) & \overline{D h(z)}
\end{array}\right), \quad z \in B^{n} .
$$

Elementary computations yield

$$
J_{f}(z)=|\operatorname{det} D h(z)|^{2} \operatorname{det}\left(I_{n}-D g(z)[D h(z)]^{-1} \overline{D g(z)[D h(z)]^{-1}}\right) .
$$

Hence $J_{f}(z) \neq 0$ for all $z \in B^{n}$, since the hypothesis ensures that 1 is not an eigenvalue of the matrix $D g(z)[D h(z)]^{-1} \overline{D g(z)[D h(z)]^{-1}}$.

Next let $f_{t}=h+t \bar{g}$, where $0 \leq t \leq 1$. Since $\left\|D g(z)[D h(z)]^{-1}\right\|<1$ for $z \in B^{n}$, it follows that $J_{f_{t}}(z) \neq 0$ for all $t \in[0,1]$ and $z \in B^{n}$, by the above argument. But $J_{f_{t}}(z)$ is continuous with respect to $t$ and $J_{f_{0}}(z)=|\operatorname{det} D h(z)|^{2}>0$ for $z \in B^{n}$, so we conclude that $J_{f}(z)>0$ for $z \in B^{n}$. In other words, the mapping $f$ is sense-preserving.

The next result provides a method for constructing examples of pluriharmonic mappings in the family $S_{H}\left(B^{n}\right)$. Recall that $K\left(B^{n}\right)$ denotes the family of biholomorphic mappings of class $S\left(B^{n}\right)$ with convex images. The following theorem is due to Mocanu 24 in the case $n=1$.

Theorem 6. Let $h \in K\left(B^{n}\right)$, and let $g \in \mathcal{H}\left(B^{n}\right)$ with $g(0)=0$. Suppose that $\left\|D g(z)[D h(z)]^{-1}\right\|<1, z \in B^{n}$. Then $f=h+\bar{g}$ is a sense-preserving mapping in $S_{H}\left(B^{n}\right)$.

Proof. Let $\psi=f \circ h^{-1}$. Then $\psi$ is pluriharmonic on the convex domain $\Omega=h\left(B^{n}\right)$ and $\psi(w)=w+\overline{\left(g \circ h^{-1}\right)(w)}$ for $w \in \Omega$. Let $w_{1}$ and $w_{2}$ be arbitrary points in $\Omega$ with $w_{1} \neq w_{2}$. Then by convexity the points $w(t)=(1-t) w_{1}+t w_{2}$ lie in $\Omega$ for $t \in[0,1]$ and we can define $z(t)=h^{-1}(w(t))$. In view of the hypothesis, we deduce that

$$
\begin{aligned}
& \operatorname{Re}\left\langle\psi\left(w_{2}\right)-\psi\left(w_{1}\right), \frac{w_{2}-w_{1}}{\left\|w_{2}-w_{1}\right\|^{2}}\right\rangle=\int_{0}^{1} \operatorname{Re}\left\langle\frac{d}{d t} \psi(w(t)), \frac{w_{2}-w_{1}}{\left\|w_{2}-w_{1}\right\|^{2}}\right\rangle d t \\
& =1+\int_{0}^{1} \operatorname{Re}\left\langle D g(z(t))[D h(z(t))]^{-1}\left(w_{2}-w_{1}\right), \frac{\overline{w_{2}}-\overline{w_{1}}}{\left\|w_{2}-w_{1}\right\|^{2}}\right\rangle d t \\
& \geq 1-\int_{0}^{1}\left\|D g(z(t))[D h(z(t))]^{-1}\right\| d t>0 .
\end{aligned}
$$

Hence $\psi\left(w_{1}\right) \neq \psi\left(w_{2}\right)$, and thus $\psi$ is univalent on $\Omega$. Then $f=\psi \circ h$ is also univalent on $B^{n}$, so that $f \in S_{H}\left(B^{n}\right)$, as claimed. The fact that $f$ is sense-preserving follows from Theorem 5 . This completes the proof. 
If $h \in S\left(B^{n}\right)$ and $A \in L\left(\mathbb{C}^{n}, \mathbb{C}^{n}\right)$ with $\|A\|<1$, it is not difficult to see that $f=h+A \bar{h} \in S_{H}\left(B^{n}\right)$. In fact, $f$ is sense-preserving, by Theorem 5. Moreover, if $h \in K\left(B^{n}\right)$, then $f$ is a convex mapping of class $S_{H}\left(B^{n}\right)$.

Examples. We pause now to give three examples of mappings $g$ and $h$ which satisfy the conditions of Theorem 6 . Assume for simplicity that $n=2$, and write $h=h(z, w)$ and $g=g(z, w)$.

(i) Let $h(z, w)=(z, w)$ and take $g$ to be an arbitrary holomorphic mapping with $g(0)=0$ and $\|D g(z, w)\|<1$ for all points $(z, w) \in B^{2}$. Then, clearly, $g$ and $h$ satisfy the conditions of Theorem 6 .

(ii) Next let $h(z, w)=(z, w) /(1-z)$ and define

$$
g(z, w)=r\left(\frac{z}{1-z}+\log (1-z), \frac{w^{2}}{2(1-z)}\right)
$$

where $r>0$. Then $h \in K\left(B^{2}\right)$, as shown for instance in [33], and

$$
D g(z, w)[D h(z, w)]^{-1}=r\left(\begin{array}{cc}
z & 0 \\
-\frac{w^{2}}{2} & w
\end{array}\right) .
$$

For any $(u, v) \in \mathbb{C}^{2}$ with $\|(u, v)\|=1$, an application of the Cauchy-Schwarz inequality to $\left|-\frac{w^{2}}{2} u+w v\right|$ gives

$$
\begin{aligned}
\left\|D g(z, w)[D h(z, w)]^{-1}(u, v)\right\|^{2} & =r^{2}\left(|z u|^{2}+\left|-\frac{w^{2}}{2} u+w v\right|^{2}\right) \\
& \leq r^{2}\left(|z|^{2}+|w|^{2}+\frac{1}{4}|w|^{4}\right)<\frac{5}{4} r^{2} .
\end{aligned}
$$

Thus $g$ and $h$ satisfy the conditions of Theorem 6 for $r \leq 2 / \sqrt{5}$.

(iii) Finally, let

$$
h(z, w)=\left(\frac{1}{2} \log \left(\frac{1+z}{1-z}\right), \frac{w}{\sqrt{1-z^{2}}}\right)
$$

and

$$
g(z, w)=r\left(-\frac{1}{2} \log \left(1-z^{2}\right), \frac{w^{2}}{2 \sqrt{1-z^{2}}}\right),
$$

where $r>0$. Then $h \in K\left(B^{2}\right)$ by a theorem of Roper and Suffridge [33, and

$$
D g(z, w)[D h(z, w)]^{-1}=r\left(\begin{array}{cc}
z & 0 \\
-\frac{z w^{2}}{2 \sqrt{1-z^{2}}} & w
\end{array}\right) .
$$

For any $(u, v) \in \mathbb{C}^{2}$ with $\|(u, v)\|=1$, another application of the Cauchy-Schwarz inequality gives

$$
\begin{aligned}
\left\|D g(z, w)[D h(z, w)]^{-1}(u, v)\right\|^{2} & =r^{2}\left(|z u|^{2}+\left|-\frac{z w^{2}}{2 \sqrt{1-z^{2}}} u+w v\right|^{2}\right) \\
& \leq r^{2}\left(|z|^{2}+\frac{1}{4}|z w|^{2}+|w|^{2}\right)<\frac{17}{16} r^{2} .
\end{aligned}
$$

Thus $g$ and $h$ satisfy the conditions of Theorem 6 when $r \leq 4 / \sqrt{17}$.

For a locally biholomorphic mapping $f$ on $B^{n}$, the Koebe transform is

$$
\mathcal{K}_{\varphi}(f)(z)=[D \varphi(0)]^{-1}[D f(\varphi(0))]^{-1}\{f(\varphi(z))-f(\varphi(0))\}, \quad z \in B^{n},
$$


where $\varphi \in \operatorname{Aut}\left(B^{n}\right)$. A family $\mathcal{F} \subset \mathcal{L} S\left(B^{n}\right)$ of normalized locally biholomorphic mappings is called a linearly invariant family if $\mathcal{K}_{\varphi}(f) \in \mathcal{F}$ for every $f \in \mathcal{F}$ and every $\varphi \in \operatorname{Aut}\left(B^{n}\right)$. Various results for linearly invariant families of locally biholomorphic mappings were obtained in [8], 9], 25, 27], 28, 29], and the references therein.

For families $\mathcal{F} \subset \mathcal{L} S_{H}\left(B^{n}\right)$ of pluriharmonic mappings we introduce the following notions of linear and affine invariance.

Linear Invariance. If $f=h+\bar{g} \in \mathcal{F}$, then for each $\varphi \in \operatorname{Aut}\left(B^{n}\right)$ the mapping

$$
F(z)=[D \varphi(0)]^{-1}[D h(\varphi(0))]^{-1}[f(\varphi(z))-f(\varphi(0))], \quad z \in B^{n},
$$

also belongs to $\mathcal{F}$.

Affine Invariance. If $f=h+\bar{g} \in \mathcal{F}$ and $A \in L\left(\mathbb{C}^{n}, \mathbb{C}^{n}\right)$ has norm $\|A\|<1$, and if $h+A g$ is locally biholomorphic on $B^{n}$, then the mapping

$$
F(z)=\left[I_{n}+A D g(0)\right]^{-1}[f(z)+A \overline{f(z)}], \quad z \in B^{n},
$$

also belongs to $\mathcal{F}$.

We now define the order of a linearly invariant family $\mathcal{F} \subset \mathcal{L} S_{H}\left(B^{n}\right)$ of pluriharmonic mappings by

$$
\alpha=\alpha(\mathcal{F})=\sup \left\{\frac{1}{2}\left\|D^{2} h(0)\right\|: f=h+\bar{g} \in \mathcal{F}\right\} .
$$

By a result of Hörmander (11], Theorem 4), an equivalent definition is

$$
\alpha(\mathcal{F})=\sup \left\{\frac{1}{2}\left\|D^{2} h(0)(w, w)\right\|: f=h+\bar{g} \in \mathcal{F},\|w\|=1\right\} .
$$

In the special case where $\mathcal{F}$ is a family of locally biholomorphic mappings, $\alpha(\mathcal{F})$ is the "norm order" as introduced by Pfaltzgraff and Suffridge 29]. Moreover, for any linearly invariant family $\mathcal{F} \subset \mathcal{L} S_{H}\left(B^{n}\right)$, the family $\mathcal{F}^{\star}=\{h: h+\bar{g} \in \mathcal{F}\}$ is a linearly invariant family of normalized locally biholomorphic mappings with norm order $\alpha\left(\mathcal{F}^{\star}\right)=\alpha(\mathcal{F})$. Since a result of Pfaltzgraff and Suffridge ([29, Theorem 3.1) says that $\alpha\left(\mathcal{F}^{\star}\right) \geq 1$, we infer that $\alpha(\mathcal{F}) \geq 1$ for every linearly invariant family $\mathcal{F} \subset \mathcal{L} S_{H}\left(B^{n}\right)$ of pluriharmonic mappings.

The families $\mathcal{L} S_{H}\left(B^{n}\right)$ and $S_{H}\left(B^{n}\right)$ are affine and linearly invariant. Since $\mathcal{L} S\left(B^{n}\right) \subset \mathcal{L} S_{H}\left(B^{n}\right)$ and $\mathcal{L} S\left(B^{n}\right)$ has infinite norm order (cf. [29]), it follows that $\alpha\left(\mathcal{L} S_{H}\left(B^{n}\right)\right)=\infty$ in dimension $n>1$. In the case of one complex variable, it can be shown that $\alpha\left(S_{H}\right)<50\left(c f\right.$. 5, p. 96), but the exact order of $S_{H}$ is not known.

The family $K_{H}\left(B^{n}\right) \subset S_{H}\left(B^{n}\right)$ of pluriharmonic mappings with convex image is affine and linearly invariant. It would be interesting to estimate $\alpha\left(K_{H}\left(B^{n}\right)\right)$ in dimension $n>1$. For $n=1$ the family $K_{H}$ of convex mappings in $S_{H}$ has order 2 .

We close this section with statements of some growth and distortion theorems for linearly invariant families of locally biholomorphic mappings on $B^{n}$. These known results will be applied in the next section to obtain growth and two-point distortion theorems for pluriharmonic mappings, by arguments similar to those employed in Section 3 for harmonic mappings in the plane. Theorems $F$ and $G$ are due to Pfaltzgraff and Suffridge [29], and Theorem H was obtained by Graham, Kohr and Pfaltzgraff [10].

Theorem F. Let $\mathcal{F} \subset \mathcal{L} S\left(B^{n}\right)$ be a linearly invariant family of norm order $\alpha<\infty$ and let $f \in \mathcal{F}$. Then

$$
\frac{(1-\|z\|)^{\alpha-1}}{(1+\|z\|)^{\alpha+1}} \leq\|D f(z)\| \leq \frac{(1+\|z\|)^{\alpha-1}}{(1-\|z\|)^{\alpha+1}}, \quad z \in B^{n} .
$$


Furthermore,

$$
\frac{(1-\|z\|)^{(2 n-1) \alpha+(n-3) / 2}}{(1+\|z\|)^{(2 n-1) \alpha-(n-3) / 2}}\|w\| \leq\|D f(z)(w)\|, \quad z \in B^{n}, \quad w \in \mathbb{C}^{n} .
$$

Theorem G. Let $\mathcal{F} \subset \mathcal{L} S\left(B^{n}\right)$ be a linearly invariant family of norm order $\alpha<\infty$ and let $f \in \mathcal{F}$. Then

$$
\|f(z)\| \leq \frac{1}{2 \alpha}\left[\left(\frac{1+\|z\|}{1-\|z\|}\right)^{\alpha}-1\right], \quad z \in B^{n} .
$$

Theorem H. Let $\mathcal{F} \subset \mathcal{L} S\left(B^{n}\right)$ be a linearly invariant family of norm order $\alpha<\infty$ and let $f \in \mathcal{F}$ be biholomorphic. Then

$$
\|f(a)-f(b)\| \geq \Psi_{n, \alpha}\left(C_{B^{n}}(a, b)\right) \max \left\{T_{f}(a), T_{f}(b)\right\}, \quad a, b \in B^{n},
$$

where

$$
\Psi_{n, \alpha}(v)=\int_{0}^{v} \frac{e^{-2(2 n-1) \alpha u}}{(\cosh u)^{n-1}} d u, \quad 0 \leq v<\infty
$$

$C_{B^{n}}(a, b)$ denotes the Carathéodory metric in $B^{n}$, and

$$
T_{f}(z)=\left(1-\|z\|^{2}\right)\left\|[D f(z)]^{-1}\right\|^{-1}, \quad z \in B^{n} .
$$

Conversely, if a locally biholomorphic mapping $f$ on $B^{n}$ satisfies the inequality (18) for all $a, b \in B^{n}$, then $f$ is biholomorphic on $B^{n}$.

The definition of the Carathéodory metric will be recalled in the next section. Other two-point distortion theorems for holomorphic mappings of the unit ball in $\mathbb{C}^{n}$ may be found in [8, [10, and [16].

\section{Distortion of Pluriharmonic MAPPings}

In this section we generalize Theorem 1 to pluriharmonic mappings on the unit ball, and we consider various particular cases and consequences. We begin with two growth theorems for affine and linearly invariant families of pluriharmonic mappings on $B^{n}$. The first result is a generalization of Theorem $\mathrm{G}$ to pluriharmonic mappings.

Theorem 7. Let $\mathcal{F} \subset \mathcal{L} S_{H}\left(B^{n}\right)$ be an affine and linearly invariant family of order $\alpha<\infty$. Let $f=h+\bar{g} \in \mathcal{F}$ and suppose that $h+A g$ is locally biholomorphic on $B^{n}$ for each $A \in L\left(\mathbb{C}^{n}, \mathbb{C}^{n}\right)$ with $\|A\|<1$. Then

$$
\|f(z)\| \leq \frac{1}{2 \alpha}(1+\|D g(0)\|)\left[\left(\frac{1+\|z\|}{1-\|z\|}\right)^{\alpha}-1\right], \quad z \in B^{n} .
$$

Proof. As remarked in the previous section, $\mathcal{F}^{\star}=\{h: h+\bar{g} \in \mathcal{F}\}$ is a linearly invariant family of normalized locally biholomorphic mappings with norm order $\alpha\left(\mathcal{F}^{\star}\right)=\alpha(\mathcal{F})$. Thus it follows from Theorem $\mathrm{F}$ that

$$
\|D h(z)\| \leq \frac{(1+\|z\|)^{\alpha-1}}{(1-\|z\|)^{\alpha+1}}, \quad z \in B^{n}
$$

whenever $f=h+\bar{g} \in \mathcal{F}$. On the other hand, in view of the hypothesis that $h+A g$ is locally biholomorphic on $B^{n}$ for $A \in L\left(\mathbb{C}^{n}, \mathbb{C}^{n}\right)$ with $\|A\|<1$, the affine invariance of the family $\mathcal{F}$ shows that

$$
\left.\left[I_{n}+A D g(0)\right]^{-1}(f+A \bar{f})=\left[I_{n}+A D g(0)\right]^{-1}[(h+A g)+(A \bar{h}+\bar{g}))\right]
$$


also belongs to the family $\mathcal{F}$. Applying Theorem $\mathrm{F}$ to its holomorphic part, we infer that

$$
\left\|\left[I_{n}+A D g(0)\right]^{-1}[D h(z)+A D g(z)]\right\| \leq \frac{(1+r)^{\alpha-1}}{(1-r)^{\alpha+1}}, \quad r=\|z\|<1 .
$$

Consequently, we find that

$$
\begin{aligned}
\|D h(z)+A D g(z)\| & \leq\left\|\mid I_{n}+A D g(0)\right\|\left\|\left[I_{n}+A D g(0)\right]^{-1}[D h(z)+A D g(z)]\right\| \\
& \leq \mu(g) \frac{(1+r)^{\alpha-1}}{(1-r)^{\alpha+1}},
\end{aligned}
$$

where $\mu(g)=\sup _{\|A\| \leq 1}\left\|I_{n}+A D g(0)\right\|$. Hence

$$
\|D h(z)(w)+A D g(z)(w)\| \leq \mu(g) \frac{(1+r)^{\alpha-1}}{(1-r)^{\alpha+1}}\|w\|, \quad w \in \mathbb{C}^{n},
$$

for all $A \in L\left(\mathbb{C}^{n}, \mathbb{C}^{n}\right)$ with $\|A\| \leq 1$. In particular, for each fixed $z \in B^{n}$ and $w \in \mathbb{C}^{n}$ we can choose $A$ to be a unitary matrix such that $A D g(z)(w)=c D h(z)(w)$ for some $c \geq 0$. Then since $\|A\|=1$ and $A$ acts isometrically on $\mathbb{C}^{n}$, we deduce that

$$
\|D h(z)(w)\|+\|D g(z)(w)\| \leq \mu(g) \frac{(1+r)^{\alpha-1}}{(1-r)^{\alpha+1}}\|w\|
$$

for all $z \in B^{n}$ and $w \in \mathbb{C}^{n}$, where $r=\|z\|<1$.

Now fix an arbitrary point $z \in B^{n}$ and define the function

$$
v(t)=\operatorname{Re}\langle f(t z), f(z)\rangle, \quad 0 \leq t \leq 1 .
$$

Then $v(0)=0$ and

$$
\begin{aligned}
\|f(z)\|^{2} & =v(1)=\int_{0}^{1} v^{\prime}(t) d t=\int_{0}^{1} \operatorname{Re}\langle D h(t z)(z)+\overline{D g(t z)(z)}, f(z)\rangle d t \\
& \leq\|f(z)\| \int_{0}^{1}(\|D h(t z)(z)\|+\|D g(t z)(z)\|) d t .
\end{aligned}
$$

Hence it follows from (20) that

$$
\begin{aligned}
\|f(z)\| & \leq \mu(g) \int_{0}^{1} \frac{(1+r t)^{\alpha-1}}{(1-r t)^{\alpha+1}} r d t \\
& =\mu(g) \frac{1}{2 \alpha}\left[\left(\frac{1+r}{1-r}\right)^{\alpha}-1\right], \quad r=\|z\|<1 .
\end{aligned}
$$

The proof is completed with the observation that $\mu(g)=1+\|D g(0)\|$. To see this, let $x$ be a unit vector such that $\|D g(0) x\|=\|D g(0)\|$ and let $A$ be a unitary matrix for which $A D g(0) x=c x$ for some $c \geq 0$. Then we have

$$
c=\|c x\|=\|A D g(0) x\|=\|D g(0) x\|=\|D g(0)\|,
$$

and so

$$
\mu(g) \geq\left\|\left[I_{n}+A D g(0)\right] x\right\|=\|(1+c) x\|=1+c=1+\|D g(0)\| .
$$

The reverse inequality is trivial.

The next theorem is a generalization to pluriharmonic mappings of a result obtained in 10 for biholomorphic mappings. The proof will appeal to the following lemma, which is implicit in the proof of the corresponding result (Theorem 5) in [10. 
Lemma 2. For $\alpha \geq 1$, let

$$
\Theta_{n, \alpha}(t)=\frac{(1-t)^{(2 n-1) \alpha+(n-3) / 2}}{(1+t)^{(2 n-1) \alpha-(n-3) / 2}}, \quad 0 \leq t \leq 1 .
$$

Then

$$
\int_{0}^{r} \Theta_{n, \alpha}(t) d t=\Psi_{n, \alpha}(\operatorname{arctanh} r), \quad 0 \leq r<1,
$$

where $\Psi_{n, \alpha}$ is defined by (19).

Theorem 8. Let $\mathcal{F} \subset S_{H}\left(B^{n}\right)$ be an affine and linearly invariant family of pluriharmonic mappings of finite order $\alpha=\alpha(\mathcal{F})$. Let $f=h+\bar{g} \in \mathcal{F}$ and suppose that $\|D g(0)\|<1$ and that $h+A g$ is locally biholomorphic on $B^{n}$ for each $A \in L\left(\mathbb{C}^{n}, \mathbb{C}^{n}\right)$ with $\|A\|<1$. Then

$$
\|f(z)\| \geq(1-\|D g(0)\|) \Psi_{n, \alpha}(\operatorname{arctanh}\|z\|), \quad z \in B^{n},
$$

where $\Psi_{n, \alpha}$ is defined by (19).

Proof. As in the proof of Theorem 7, we apply Theorem F and the affine invariance of the family $\mathcal{F}$ to conclude that

$\left\|\left[I_{n}+A D g(0)\right]^{-1}[D h(z)+A D g(z)] w\right\| \geq \Theta_{n, \alpha}(r)\|w\|, \quad r=\|z\|<1, \quad w \in \mathbb{C}^{n}$, for all $A \in L\left(\mathbb{C}^{n}, \mathbb{C}^{n}\right)$ with $\|A\|<1$, where $\Theta_{n, \alpha}$ is defined by (21). It follows that

$$
\|D h(z)(w)+A D g(z)(w)\| \geq \lambda(g) \Theta_{n, \alpha}(r)\|w\|, \quad z \in B^{n}, \quad w \in \mathbb{C}^{n},
$$

where $\lambda(g)=\inf _{\|A\| \leq 1}\left\|\left[I_{n}+A D g(0)\right]^{-1}\right\|^{-1}$. Next, for fixed $z \in B^{n}$ and $w \in \mathbb{C}^{n}$, choose $A$ to be a unitary matrix such that

$$
A D g(z)(w)=-c D h(z)(w) \quad \text { for some } c \geq 0 .
$$

This choice of $A$ gives the inequality

$$
|\|D h(z)(w)\|-\|D g(z)(w)\|| \geq \lambda(g) \Theta_{n, \alpha}(r)\|w\|
$$

for all $z \in B^{n}$ and $w \in \mathbb{C}^{n}$, where $r=\|z\|$.

For $r \in(0,1)$, let $m(r)=\min \{\|f(z)\|:\|z\|=r\}$ and choose a point $z_{0} \in \partial B_{r}^{n}$ where $\left\|f\left(z_{0}\right)\right\|=m(r)$. Then the linear segment

$$
\Gamma=\left\{t f\left(z_{0}\right): 0 \leq t \leq 1\right\}
$$

has the property $\Gamma \subset \overline{B_{m(r)}^{n}} \subset f\left(\overline{B_{r}^{n}}\right)$, which shows that $\gamma=f^{-1}(\Gamma) \subset \overline{B_{r}^{n}}$. Now integrate with respect to arclength and apply (22) to obtain

$$
\begin{aligned}
m(r) & =\left\|f\left(z_{0}\right)\right\|=\int_{\Gamma}\|d w\|=\int_{\gamma}\|D h(\zeta) d \zeta+\overline{D g(\zeta)} d \bar{\zeta}\| \\
& \geq \int_{\gamma}|\|D h(\zeta) d \zeta\|-\|D g(\zeta) d \zeta\|| \geq \lambda(g) \int_{0}^{r} \Theta_{n, \alpha}(\|\zeta\|) d\|\zeta\| .
\end{aligned}
$$

Thus in view of Lemma 2, we conclude that

$$
\|f(z)\| \geq m(\|z\|) \geq \lambda(g) \Psi_{n, \alpha}(\operatorname{arctanh}\|z\|), \quad z \in B^{n} .
$$

To complete the proof, we observe that $\lambda(g)=1-\|D g(0)\|$. Since $\|A D g(0)\| \leq$ $\|D g(0)\|<1$, a Neumann series development of $\left[I_{n}+A D g(0)\right]^{-1}$ shows that $\lambda(g) \geq$ $1-\|D g(0)\|$. For the reverse inequality, let $x$ be a unit vector such that $\|D g(0) x\|=$ 
$\|D g(0)\|$ and let $A$ be a unitary matrix such that $A D g(0) x=-c x$ for some $c \geq 0$. Also, let $y=\left[I_{n}+A D g(0)\right] x$. Then

$$
\begin{aligned}
& c=\|c x\|=\|-A D g(0) x\|=\|D g(0) x\|=\|D g(0)\| \quad \text { and } \\
& \|y\|=\left\|\left[I_{n}+A D g(0)\right] x\right\|=\|(1-c) x\|=1-c, \quad \text { so that } \\
1= & \|x\|=\left\|\left[I_{n}+A D g(0)\right]^{-1} y\right\| \leq\left\|\left[I_{n}+A D g(0)\right]^{-1}\right\| \cdot\|y\| \\
= & \left\|\left[I_{n}+A D g(0)\right]^{-1}\right\|(1-c) .
\end{aligned}
$$

Therefore,

$$
\lambda(g) \leq\left\|\left[I_{n}+A D g(0)\right]^{-1}\right\|^{-1} \leq 1-c=1-\|D g(0)\| .
$$

This completes the proof of Theorem 8 .

The next theorem is a generalization of Theorem 1 and Theorem $\mathrm{H}$ to pluriharmonic mappings of the unit ball. It may be viewed as an invariant form of Theorem 8. Like Theorem 1, it provides a necessary and sufficient condition for univalence of a large class of pluriharmonic mappings.

Recall that the Carathéodory metric in the unit ball $B^{n}$ is given by

$$
C_{B^{n}}(a, b)=\frac{1}{2} \log \frac{1+\left\|\varphi_{a}^{-1}(b)\right\|}{1-\left\|\varphi_{a}^{-1}(b)\right\|},
$$

where $\varphi_{a}(z)$ is the biholomorphic automorphism of $B^{n}$ defined by

$$
\varphi_{a}(z)=\Lambda_{a}\left(\frac{z+a}{1+\langle z, a\rangle}\right), \quad z \in B^{n},
$$

and $\Lambda_{a}$ is the linear operator

$$
\Lambda_{a}(w)=\frac{\langle w, a\rangle}{1+s_{a}} a+s_{a} w, \quad w \in \mathbb{C}^{n},
$$

with $s_{a}=\sqrt{1-\|a\|^{2}}$. Then $\varphi_{a}(-a)=0, \varphi_{a}(0)=a$, and $\varphi_{0}(z)=z$ for all $z \in B^{n}$. The Carathéodory metric is invariant under biholomorphic automorphisms of $B^{n}$. In particular, $C_{B^{n}}\left(\varphi_{a}(z), \varphi_{a}(w)\right)=C_{B^{n}}(z, w)$ for each $a \in B^{n}$ and all $z, w \in B^{n}$. For the unit ball the Carathéodory metric coincides with other metrics such as the Bergman and Kobayashi metrics. For $n=1$ it reduces to the hyperbolic metric in the unit disk, as defined in Section 1. For further information, see [6, 12, 17, and 36.

It is convenient to also introduce the special notation

$$
S(z)=\left(1-\|z\|^{2}\right)\left\|[D h(z)]^{-1}\right\|^{-1}\left(1-\left\|D g(z)[D h(z)]^{-1}\right\|\right), \quad z \in B^{n},
$$

and

$$
\nu(g)=\inf _{\|A\| \leq 1}\left\{\frac{1}{\left\|\left[I_{n}+A D g(0)\right]^{-1}\right\| \cdot\left\|I_{n}+A D g(0)\right\|}\right\},
$$

where $g \in \mathcal{H}\left(B^{n}\right)$ with $\|D g(0)\|<1$ and $h$ is locally biholomorphic on $B^{n}$. Note that $\nu(g) \geq(1-\|D g(0)\|) /(1+\|D g(0)\|)$.

Theorem 9. Let $\mathcal{F} \subset \mathcal{L} S_{H}\left(B^{n}\right)$ be an affine and linearly invariant family of pluriharmonic mappings of order $\alpha=\alpha(\mathcal{F})<\infty$, and let $f=h+\bar{g} \in \mathcal{F}$. Suppose that $h+A g$ is biholomorphic on $B^{n}$ for each $A \in L\left(\mathbb{C}^{n}, \mathbb{C}^{n}\right)$ with $\|A\|<1$, and that

$$
\left\|D g(z)[D h(z)]^{-1}\right\|<1, \quad z \in B^{n} .
$$


Then

$$
\|f(a)-f(b)\| \geq \Phi_{n, \alpha}(a, b), \quad a, b \in B^{n},
$$

where

$$
\Phi_{n, \alpha}(a, b)=\nu(g) \Psi_{n, \alpha}\left(C_{B^{n}}(a, b)\right) \max \{S(a), S(b)\}
$$

and $\Psi_{n, \alpha}$ is defined by (19). Conversely, if $f=h+\bar{g}$ is a pluriharmonic mapping that has the property (24) and satisfies (25) for some $\alpha>0$ and all $a, b \in B^{n}$, and if $h$ is locally biholomorphic and $\|D g(0)\|<1$, then $f$ is univalent on $B^{n}$.

Proof. Again we observe that $\mathcal{F}^{\star}=\{h: h+\bar{g} \in \mathcal{F}\}$ is a linearly invariant family of locally biholomorhic mappings on $B^{n}$ with norm order $\alpha=\alpha(\mathcal{F})$. If $f=h+\bar{g} \in \mathcal{F}$, then by hypothesis, $h+A g$ is biholomorphic on $B^{n}$ for each $A \in L\left(\mathbb{C}^{n}, \mathbb{C}^{n}\right)$ with $\|A\|<1$, so by affine invariance the mapping

$$
\begin{aligned}
F & =H+\bar{G}=\left[I_{n}+A D g(0)\right]^{-1}[f+A \bar{f}] \\
& =\left[I_{n}+A D g(0)\right]^{-1}[(h+A g)+(A \bar{h}+\bar{g})]
\end{aligned}
$$

also belongs to $\mathcal{F}$. Therefore, the mapping $H=\left[I_{n}+A D g(0)\right]^{-1}(h+A g)$ belongs to $\mathcal{F}^{\star}$ and is biholomorphic, and so by Theorem $\mathrm{H}$ it has the two-point distortion property (18). In other words,

$$
\begin{aligned}
& \left\|\left[I_{n}+A D g(0)\right]^{-1}[(h(a)-h(b))+A(g(a)-g(b))]\right\| \\
\geq & \Psi_{n, \alpha}\left(C_{B^{n}}(a, b)\right) \max \left\{T_{H}(a), T_{H}(b)\right\}, \quad a, b \in B^{n},
\end{aligned}
$$

where

$$
T_{H}(z)=\left(1-\|z\|^{2}\right)\left\|[D H(z)]^{-1}\right\|^{-1}, \quad z \in B^{n} .
$$

On the other hand, by (24) and the fact that $\|A\|<1$, we see that

$$
\left\|\left(I_{n}+A D g(z)[D h(z)]^{-1}\right)^{-1}\right\| \leq \frac{1}{1-\left\|D g(z)[D h(z)]^{-1}\right\|}, \quad z \in B^{n},
$$

and so we find after some manipulation that

$$
T_{H}(z) \geq \frac{S(z)}{\left\|I_{n}+A D g(0)\right\|}, \quad z \in B^{n}, \quad\|A\|<1 .
$$

Combining (27) and (28), we conclude that

$$
\|(h(a)-h(b))+A(g(a)-g(b))\| \geq \Phi_{n, \alpha}(a, b), \quad a, b \in B^{n},
$$

for all $A \in L\left(\mathbb{C}^{n}, \mathbb{C}^{n}\right)$ with $\|A\| \leq 1$. Finally, for arbitrary fixed $a, b \in B^{n}$, choose $A$ to be a unitary matrix for which

$$
A(g(a)-g(b))=-c(h(a)-h(b)) \quad \text { for some } c \geq 0 .
$$

It then follows from (29) that

$$
\|f(a)-f(b)\| \geq|\|h(a)-h(b)\|-\|g(a)-g(b)\|| \geq \Phi_{n, \alpha}(a, b), \quad a, b \in B^{n},
$$

which is the inequality (25).

Conversely, let $f=h+\bar{g}$ be a pluriharmonic mapping with $h$ locally biholomorphic and $\|D g(0)\|<1$. Suppose that $f$ has the property (24) and satisfies (25) for some $\alpha>0$ and all $a, b \in B^{n}$. Then if $f(a)=f(b)$ for some $a, b \in B^{n}$, it must follow that $C_{B^{n}}(a, b)=0$, so that $a=b$. This shows that $f$ is univalent in $B^{n}$. 
Taking Theorems 5 and 6 into account, we obtain two corollaries of Theorem 9 that provide sufficient conditions for sense-preserving univalence of pluriharmonic mappings.

Corollary 1. Let $\mathcal{F} \subset \mathcal{L} S_{H}\left(B^{n}\right)$ be an affine and linearly invariant family of pluriharmonic mappings of order $\alpha=\alpha(\mathcal{F})<\infty$, and let $f=h+\bar{g} \in \mathcal{F}$. Suppose that $h+A g$ is biholomorphic on $B^{n}$ for each $A \in L\left(\mathbb{C}^{n}, \mathbb{C}^{n}\right)$ with $\|A\|<1$, and that the condition (24) holds. Then $f$ is univalent and sense-preserving in $B^{n}$ and it has the two-point distortion property (25).

Corollary 2. Let $\mathcal{F} \subset \mathcal{L} S_{H}\left(B^{n}\right)$ be an affine and linearly invariant family of pluriharmonic mappings of order $\alpha=\alpha(\mathcal{F})<\infty$, and let $f=h+\bar{g} \in \mathcal{F}$. Suppose that $h \in K\left(B^{n}\right)$ and that the condition (24) holds. Then $f$ is univalent and sensepreserving in $B^{n}$ and it has the property (25).

Proof of Corollary 2. By an argument similar to that in the proof of Theorem 6, we may infer that if $h \in K\left(B^{n}\right)$ and $g \in \mathcal{H}\left(B^{n}\right)$, and if they satisfy the condition (24), then $h+A g$ is biholomorphic on $B^{n}$ for each $A \in L\left(\mathbb{C}^{n}, \mathbb{C}^{n}\right)$ with $\|A\|<1$. In fact, $h+A g$ is close-to-convex with respect to $h$ (see [38]). The result now follows from Corollary 1.

The next theorem complements Theorem 9 by providing an upper bound for the distortion $\|f(a)-f(b)\|$. It generalizes the estimate (9) of Theorem 1 to pluriharmonic mappings on $B^{n}$. Before stating the theorem, it will be convenient to introduce some additional notation.

For $g, h \in \mathcal{H}\left(B^{n}\right)$, let

$$
\widetilde{S}(a)=\left\|D \varphi_{a}(0)\right\|(\|D h(a)\|+\|D g(a)\|), \quad a \in B^{n},
$$

where $\varphi_{a}$ is the biholomorphic automorphism of $B^{n}$ defined by (23). Clearly, $\left|\varphi_{a}^{\prime}(0)\right|=1-|a|^{2}$ if $n=1$. For $n \geq 2$ it is known (cf. Rudin [36]) that

$$
1-\|a\|^{2} \leq\left\|D \varphi_{a}(0)\right\| \leq \sqrt{1-\|a\|^{2}}, \quad a \in B^{n} .
$$

Therefore,

$$
\widetilde{S}(a) \leq \sqrt{1-\|a\|^{2}}(\|D h(a)\|+\|D g(a)\|), \quad a \in B^{n} .
$$

We also introduce the notation

$$
\chi(g)=\sup _{\|A\| \leq 1}\left\{\left\|\left[I_{n}+A D g(0)\right]^{-1}\right\| \cdot\left\|I_{n}+A D g(0)\right\|\right\}
$$

for $g \in \mathcal{H}\left(B^{n}\right)$ with $\|D g(0)\|<1$. Note that $\chi(g) \leq(1+\|D g(0)\|) /(1-\|D g(0)\|)$.

Theorem 10. Let $\mathcal{F} \subset \mathcal{L} S_{H}\left(B^{n}\right)$ be an affine and linearly invariant family of pluriharmonic mappings of order $\alpha=\alpha(\mathcal{F})<\infty$. Let $f=h+\bar{g}$ be a mapping in the family $\mathcal{F}$ with the property (24). Then for arbitrary $a, b \in B^{n}$,

$$
\|f(a)-f(b)\| \leq \chi(g) \frac{1}{2 \alpha}\left[\exp \left(2 \alpha C_{B^{n}}(a, b)\right)-1\right] \min \{\widetilde{S}(a), \widetilde{S}(b)\} .
$$

Proof. We begin by showing that if $f=h+\bar{g} \in \mathcal{F}$, then

$$
\|h(a)-h(b)\| \leq \frac{1}{2 \alpha}\left[\exp \left(2 \alpha C_{B^{n}}(a, b)\right)-1\right] \min \left\{\widetilde{T}_{h}(a), \widetilde{T}_{h}(b)\right\}
$$

for all $a, b \in B^{n}$, where

$$
\widetilde{T}_{h}(z)=\left\|D \varphi_{z}(0)\right\| \cdot\|D h(z)\|, \quad z \in B^{n},
$$


and $\varphi_{z}$ is the biholomorphic automorphism of $B^{n}$ given by (23). Indeed, since $h$ belongs to the linearly invariant family $\mathcal{F}^{\star}=\{h: h+\bar{g} \in \mathcal{F}\}$ of locally biholomorphic mappings with norm order $\alpha\left(\mathcal{F}^{\star}\right)=\alpha(\mathcal{F})$, it follows from Theorem $\mathrm{G}$ that

$$
\|h(z)\| \leq \frac{1}{2 \alpha}\left\{\left(\frac{1+\|z\|}{1-\|z\|}\right)^{\alpha}-1\right\}, \quad z \in B^{n} .
$$

By the definition of the Carathéodory metric, this inequality takes the equivalent form

$$
\|h(z)\| \leq \frac{1}{2 \alpha}\left[\exp \left(2 \alpha C_{B^{n}}(0, z)\right)-1\right], \quad z \in B^{n} .
$$

But for each fixed $a \in B^{n}$ the mapping

$$
\widetilde{h}(z)=\left[D \varphi_{a}(0)\right]^{-1}[D h(a)]^{-1}\left[h\left(\varphi_{a}(z)\right)-h(a)\right]
$$

also belongs to $\mathcal{F}^{\star}$, because of the linear invariance, so that

$$
\|\widetilde{h}(z)\| \leq \frac{1}{2 \alpha}\left[\exp \left(2 \alpha C_{B^{n}}(0, z)\right)-1\right], \quad z \in B^{n} .
$$

Now let $b=\varphi_{a}(z)$ and observe that

$$
C_{B^{n}}(0, z)=C_{B^{n}}\left(\varphi_{a}^{-1}(a), \varphi_{a}^{-1}(b)\right)=C_{B^{n}}(a, b)
$$

by invariance of the Carathéodory metric. Consequently,

$$
\begin{aligned}
\|h(a)-h(b)\| & \leq\|D h(a)\|\left\|D \varphi_{a}(0)\right\|\left\|\widetilde{h}\left(\varphi_{a}^{-1}(b)\right)\right\| \\
& \leq\|D h(a)\|\left\|D \varphi_{a}(0)\right\| \frac{1}{2 \alpha}\left[\exp \left(2 \alpha C_{B^{n}}(a, b)\right)-1\right] \\
& \leq \frac{1}{2 \alpha}\left[\exp \left(2 \alpha C_{B^{n}}(a, b)\right)-1\right] \widetilde{T}_{h}(a) .
\end{aligned}
$$

Interchanging the roles of $a$ and $b$, we arrive at the inequality (31).

Since $\mathcal{F} \subset \mathcal{L} S_{H}\left(B^{n}\right)$, it follows from Theorem 5 that for any $f=h+\bar{g} \in \mathcal{F}$ with the property (24), the mapping $h+A g$ is locally biholomorphic in $B^{n}$ for each $A \in L\left(\mathbb{C}^{n}, \mathbb{C}^{n}\right)$ with $\|A\|<1$. Therefore, by the affine invariance of $\mathcal{F}$, the mapping

$$
F(z)=\left[I_{n}+A D g(0)\right]^{-1}[f(z)+A \overline{f(z)}]
$$

also belongs to $\mathcal{F}$ for $\|A\|<1$. Writing $F=H+\bar{G}$, we conclude from (31) that

$$
\|H(a)-H(b)\| \leq \frac{1}{2 \alpha}\left[\exp \left(2 \alpha C_{B^{n}}(a, b)\right)-1\right] \min \left\{\widetilde{T}_{H}(a), \widetilde{T}_{H}(b)\right\}
$$

for all $a, b \in B^{n}$. But a simple calculation shows that

$$
H(z)=\left[I_{n}+A D g(0)\right]^{-1}[h(z)+A g(z)],
$$

which easily gives the estimate

$$
\widetilde{T}_{H}(z) \leq \widetilde{S}(z)\left\|\left[I_{n}+A D g(0)\right]^{-1}\right\|, \quad z \in B^{n} .
$$

Combining this with (33), we infer that

$$
\begin{aligned}
\| h(a)-h(b) & +A(g(a)-g(b)) \| \\
& \leq \chi(g) \frac{1}{2 \alpha}\left[\exp \left(2 \alpha C_{B^{n}}(a, b)\right)-1\right] \min \{\widetilde{S}(a), \widetilde{S}(b)\}
\end{aligned}
$$

for each $A \in L\left(\mathbb{C}^{n}, \mathbb{C}^{n}\right)$ with $\|A\| \leq 1$ and for all $a, b \in B^{n}$. 
Finally, fix arbitrary points $a, b \in B^{n}$. If $g(a)=g(b)$, then the inequality (34) reduces to (30) and the proof is finished. If $g(a) \neq g(b)$ and $h(a) \neq h(b)$, choose $A$ to be a unitary matrix for which

$$
A(g(a)-g(b))=c(h(a)-h(b)) \quad \text { for some } c>0 .
$$

If $h(a)=h(b)$, choose $A$ to be an arbitrary unitary matrix. Then in either case it follows from (34) that

$$
\begin{aligned}
\|f(a)-f(b)\| & \leq\|h(a)-h(b)\|+\|g(a)-g(b)\| \\
& \leq \chi(g) \frac{1}{2 \alpha}\left[\exp \left(2 \alpha C_{B^{n}}(a, b)\right)-1\right] \min \{\widetilde{S}(a), \widetilde{S}(b)\},
\end{aligned}
$$

as claimed. This completes the proof.

In view of Theorems 9,10 , and 5 , we obtain the following consequence. For any family $\mathcal{F} \subset \mathcal{L} S_{H}\left(B^{n}\right)$, we denote by $\mathcal{F}^{0}$ the subfamily of mappings $f=h+\bar{g} \in \mathcal{F}$ for which $D g(0)=0$.

Corollary. Let $\mathcal{F} \subset \mathcal{L} S_{H}\left(B^{n}\right)$ be an affine and linearly invariant family of pluriharmonic mappings of order $\alpha=\alpha(\mathcal{F})<\infty$ and let $f=h+\bar{g} \in \mathcal{F}^{0}$. If the condition (24) holds, then

$$
\|f(a)-f(b)\| \leq \frac{1}{2 \alpha}\left[\exp \left(2 \alpha C_{B^{n}}(a, b)\right)-1\right] \min \{\widetilde{S}(a), \widetilde{S}(b)\}
$$

for all $a, b \in B^{n}$. Also, if $h+A g$ is biholomorphic on $B^{n}$ for each $A \in L\left(\mathbb{C}^{n}, \mathbb{C}^{n}\right)$ with $\|A\|<1$, then $f$ is a sense-preserving univalent mapping of $B^{n}$ and

$$
\|f(a)-f(b)\| \geq \Psi_{n, \alpha}\left(C_{B^{n}}(a, b)\right) \max \{S(a), S(b)\}
$$

for all $a, b \in B^{n}$, where $\Psi_{n, \alpha}$ is given by (19). Conversely, let $f=h+\bar{g}$ be a pluriharmonic mapping of $B^{n}$, where $h$ is locally biholomorphic and $g$ is holomorphic. If $f$ has the property (24) and satisfies (36) for some $\alpha>0$ and all $a, b \in B^{n}$, then $f$ is univalent and sense-preserving on $B^{n}$.

This result may be regarded as an analogue of Theorem $\mathrm{H}$ for affine and linearly invariant families of pluriharmonic mappings of $B^{n}$. In view of Theorem 1 and Theorem H, it seems plausible that the lower bound (36) can be sharpened as follows.

Conjecture. Let $\mathcal{F} \subset S_{H}\left(B^{n}\right)$ be an affine and linearly invariant family of pluriharmonic mappings of order $\alpha=\alpha(\mathcal{F})<\infty$ and let $f=h+\bar{g} \in \mathcal{F}^{0}$. If $f$ satisfies the condition (24), then

$$
\|f(a)-f(b)\| \geq \frac{1}{2 \alpha}\left[1-\exp \left(-2 \alpha C_{B^{n}}(a, b)\right)\right] \max \{S(a), S(b)\}, \quad a, b \in B^{n} .
$$

\section{ACKNOWLEDGEMENTS}

Some of the research for this paper was carried out in May 2009 while Gabriela Kohr visited the Department of Mathematics at the University of Toronto. She expresses her gratitude to members of that department for their hospitality during the visit.

The authors thank the referee for a careful reading of the paper and for suggesting the examples following Theorem 6 . 


\section{REFERENCES}

1. C. Blatter, Ein Verzerrungssatz für schlichte Funktionen, Comment. Math. Helv. 53 (1978), 651-659. MR511855 (80d:30010)

2. M. Chuaqui, P. Duren, and B. Osgood, Two-point distortion theorems for harmonic mappings, Illinois J. Math. 53 (2009), 1061-1075. MR2741178

3. J. Clunie and T. Sheil-Small, Harmonic univalent functions, Ann. Acad. Sci. Fenn. Ser. A.I 9 (1984), 3-25. MR752388 (85i:30014)

4. P. L. Duren, Univalent Functions, Springer-Verlag, New York, 1983. MR.708494 (85j:30034)

5. P. Duren, Harmonic Mappings in the Plane, Cambridge University Press, Cambridge, U. K., 2004. MR2048384 (2005d:31001)

6. P. Duren and R. Weir, The pseudohyperbolic metric and Bergman spaces in the unit ball, Trans. Amer. Math. Soc. 359 (2007), 63-76. MR2247882 (2007k:32005)

7. K. Fan, Distortion of univalent functions, J. Math. Anal. Appl. 66 (1978), 626-631. MR517751 (80a:30015)

8. S. Gong, Convex and Starlike Mappings in Several Complex Variables, Kluwer Acad. Publ., Dordrecht, 1998. MR 1689825 (2000c:32054)

9. I. Graham and G. Kohr, Geometric Function Theory in One and Higher Dimensions, Marcel Dekker Inc., New York, 2003. MR2017933 (2004i:32002)

10. I. Graham, G. Kohr and J. Pfaltzgraff, Growth and two-point distortion for biholomorphic mappings of the ball, Complex Var. Elliptic Equ. 52 (2007), 211-223. MR2297771 (2008a:32016)

11. L. Hörmander, On a theorem of Grace, Math. Scand. 2 (1954), 55-64. MR0062844 (16:27b)

12. M. Jarnicki and P. Pflug, Invariant Distances and Metrics in Complex Analysis, Walter de Gruyter \& Co., Berlin-New York, 1993. MR.1242120 (94k:32039)

13. J. A. Jenkins, On weighted distortion in conformal mapping II, Bull. London Math. Soc. 30 (1998), 151-158. MR1489326 (98m:30038)

14. J. A. Jenkins, On two point distortion theorems for bounded univalent regular functions, Kodai Math. J. 24 (2001), 329-338. MR1866369 (2002h:30012)

15. S. A. Kim and D. Minda, Two-point distortion theorems for univalent functions, Pacific J. Math. 163 (1994), 137-157. MR.1256180 (94m:30042)

16. G. Kohr, On some distortion results for convex mappings in $\mathbb{C}^{n}$, Complex Variables Theory Appl. 39 (1999), 161-175. MR1717651 (2000f:32018)

17. S. G. Krantz, Function Theory of Several Complex Variables, Reprint of the 1992 Edition, AMS Chelsea Publishing, Providence, RI, 2001. MR.1846625 (2002e:32001)

18. D. Kraus and O. Roth, Weighted distortion in conformal mapping in Euclidean, hyperbolic and elliptic geometry, Ann. Acad. Sci. Fenn. Math. 31 (2006), 111-130. MR2210112 (2007j:30013)

19. H. Lewy, On the non-vanishing of the Jacobian in certain one-to-one mappings, Bull. Amer. Math. Soc. 42 (1936), 689-692. MR.1563404

20. W. Ma and D. Minda, Two-point distortion theorems for bounded univalent functions, Ann. Acad. Sci. Fenn. Math. 22 (1997), 425-444. MR.1469801 (98i:30011)

21. W. Ma and D. Minda, Two-point distortion theorems for strongly close-to-convex functions, Complex Variables Theory Appl. 33 (1997), 185-205. MR1624935 (99b:30019)

22. W. Ma and D. Minda, Two-point distortion for univalent functions, J. Comput. Appl. Math. 105 (1999), 385-392. MR1690605 (2000e:30029)

23. A. W. Marshall, I. Olkin and F. Proschan, Monotonicity of ratios of means and other applications of majorization, In: Inequalities (O. Shisha, editor), Academic Press, New York, 1967, pp. 177-190. MR0237727 (38:6008)

24. P. T. Mocanu, Sufficient conditions of univalency for complex functions in the class $C^{1}$, Anal. Numer. Theor. Approx. 10 (1981), 75-79. MR670636 (84a:30025)

25. J. A. Pfaltzgraff, Distortion of locally biholomorphic maps of the $n$-ball, Complex Variables Theory Appl. 33 (1997), 239-253. MR1624947(99a:32030)

26. J. A. Pfaltzgraff, Koebe transforms of holomorphic and harmonic mappings, Talk presented at conference in Lexington, Kentucky, May 2008.

27. J. A. Pfaltzgraff and T. J. Suffridge, An extension theorem and linear invariant families generated by starlike maps, Ann. Univ. Mariae Curie-Sklodowska Sect. A 53 (1999), 193-207. MR:1778828(2001h:32023) 
28. J. A. Pfaltzgraff and T. J. Suffridge, Linear invariance, order and convex maps in $\mathbb{C}^{n}$, Complex Variables Theory Appl., vol. 40, 1999, pp. 35-50. MR1742869 (2000i:32026)

29. J. A. Pfaltzgraff and T. J. Suffridge, Norm order and geometric properties of holomorphic mappings in $\mathbf{C}^{n}$, J. Analyse Math. 82 (2000), 285-313. MR.1799667 (2001k:32028)

30. Ch. Pommerenke, Linear-invariante Familien analytischer Funktionen I, Math. Ann. 155 (1964), 108-154. MR1513275

31. Ch. Pommerenke, Univalent Functions, Vandenhoeck \& Ruprecht, Göttingen, 1975. MR0507768 (58:22526)

32. Ch. Pommerenke, Boundary Behaviour of Conformal Maps, Springer-Verlag, Berlin, 1992. MR.1217706 (95b:30008)

33. K. A. Roper and T. J. Suffridge, Convex mappings on the unit ball of $\mathbf{C}^{n}$, J. Analyse Math. 65 (1995), 333-347. MR1335379 (96m:32023)

34. O. Roth, A distortion theorem for bounded univalent functions, Ann. Acad. Sci. Fenn. Math. 27 (2002), 257-272. MR1921307 (2003j:30040)

35. O. Roth, Distortion theorems for bounded univalent functions, Analysis 23 (2003), 347-369. MR 2052374 (2005a:30024)

36. W. Rudin, Function Theory in the Unit Ball of $\mathbb{C}^{n}$, Springer-Verlag, New York, 1980. MR601594 (82i:32002)

37. T. Sheil-Small, Constants for planar harmonic mappings, J. London Math. Soc. 42 (1990), 237-248. MR1083443 (91k:30052)

38. T. J. Suffridge, Starlikeness, convexity and other geometric properties of holomorphic maps in higher dimensions, Lecture Notes in Math. Vol. 599, Springer-Verlag, 1977, 146-159. MR0450601 (56:8894)

Department of Mathematics, University of Michigan, Ann Arbor, Michigan 481091043

E-mail address: duren@umich.edu

Faculty of Engineering, Kyushu Sangyo University, 3-1 Matsukadai 2-Chome, HigashiKU FUKUOKA 813-8503, JAPAN

E-mail address: h.hamada@ip.kyusan-u.ac.jp

Faculty of Mathematics and Computer Science, Babeş-Bolyai University, 1 M. KogăLNiceanu Str., 400084 Cluj-Napoca, Romania

E-mail address: gkohr@math.ubbcluj.ro 